Revue d'histoire de l'Amérique française

MAV REVUE D.HISTOIRE DE L'AMÉRIQUE FRANÇAISE

\title{
E. P. Thompson dans des arpents de neige : les historiens canadiens-anglais et la classe ouvrière
}

\section{Desmond Morton}

Volume 37, numéro 2, septembre 1983

Travailleurs et mouvements sociaux

URI : https://id.erudit.org/iderudit/304152ar

DOI : https://doi.org/10.7202/304152ar

Aller au sommaire du numéro

Éditeur(s)

Institut d'histoire de l'Amérique française

ISSN

0035-2357 (imprimé)

1492-1383 (numérique)

Découvrir la revue

Citer cet article

Morton, D. (1983). E. P. Thompson dans des arpents de neige : les historiens canadiens-anglais et la classe ouvrière. Revue d'histoire de l'Amérique française, 37(2), 165-184. https://doi.org/10.7202/304152ar d'utilisation que vous pouvez consulter en ligne. 


\title{
E.P. THOMPSON DANS DES ARPENTS DE NEIGE: LES HISTORIENS CANADIENS-ANGLAIS ET LA CLASSE OUVRIËRE*
}

\author{
DESMOND MORTON \\ Erindale College et Centre \\ des relations industrielles \\ Université de Toronto
}

Au cours de la dernière décennie, l'historiographie du mouvement ouvrier canadien a connu une croissance rapide et s'est développée de façon considérable. De façon à la fois prévisible et inattendue, nos connaissances se sont accrues pour être aussitôt dépassées par notre curiosité. L'éventail des questions importantes dans l'histoire du mouvement ouvrier s'est élargi au point où nous ne sommes plus capables de répondre aux nouveaux besoins qui ont surgi. Simultanément, comme dans tout phénomène de croissance rapide, l'historiographie du mouvement ouvrier court le risque de se développer de façon démesurée ou de sombrer dans la stérilité ${ }^{\text {. }}$.

Il existe peu de domaines de l'historiographie contemporaine au Canada, où des progrès ont été réalisés à peu près simultanément et de façon aussi étroite chez les historiens anglophones et chez les historiens francophones. En 1972, apparaissaient à peu près au même moment le Committee on Canadian Labour History (CCLH) et le Regroupement des chercheurs en histoire des travailleurs québécois. Chacun des deux organismes s'est engagé de façon autonome dans des recherches bibliographiques et archivistiques et dans des projets de publication; dans quelques cas, il y eut même collaboration fructueuse.

Le professeur Stanley Bréhaut Ryerson a inspiré chacun de ces groupes. Au Canada, il a lancé de façon magistrale l'historiographie marxiste et, au sein de notre profession aux horizons trop étroits, il nous a rappelé de façon tenace la présence d'influences internationales et d'interprétations historiques différentes des nôtres. Entre les mains

* Conférence prononcée au Congrès de l'Institut d'histoire de l'Amérique française à l'Université du Québec à Montréal,le 22 octobre 1982.

Pour des comptes rendus, voir D.J. Bercuson, «Recent publications in Canadian Labour History», History and Social Science Teacher, XIV, 3 (printemps 1979). Pour une critique différente sur le débat historiographique, voir: G.S. Kealey, «Labour and Working-Class History in Canada: Prospects in the 1980's», Labour/Le Travailleur, VII (printemps 1981); Kenneth McNaught; «E.P. Thompson vs. Harold Logan, Writing About Labour and the Left in the 1970s», Canadian Historical Review, LXIII, 2 (juin 1981). Toutefois, on n'aborde pas du tout le sujet dans l'article de critique de H.J. Hanham «Canadian History in the 1970s», ibid., LVIII, 1 (mars 1977). 
d'un historien aussi humain, érudit et charmant, l'analyse marxiste a servi de ciselet, non de massue ${ }^{2}$.

Je ne puis d'aucune façon comparer mes compétences pour examiner l'histoire du mouvement ouvrier au Canada anglais à celles du professeur Ryerson, et j'imagine bien qu'autant votre invitation a dû étonner mes amis, autant elle a dû consterner ceux qui sont en désaccord avec mes positions historiographiques. J'ai bien sûr accepté de faire cette communication parce qu'une invitation de rendre hommage à Stanley Ryerson ne se refuse pas. Je justifie ma présence par la prétention que, règle générale, les historiens sont étrangers à ce qu'ils décrivent. Mais je ne suis pas complètement «pur». Mes travaux sur les relations politico-militaires et les conflits sociaux m'ont en effet donné la témérité, à défaut de l'intelligence, de synthétiser l'histoire du mouvement ouvrier canadien en un seul volume. L'on pourrait comparer mon expérience aux exploits de la pauvre Éliza dans Uncle Tom's Cabin, qui, dans sa course à travers le Mississipi, saute d'un banc de glace à l'autre. J'en suis sorti trempé, mais reconnaissant envers tous ceux qui m'ont fourni, ne serait-ce qu'un livre ou un article sur lequel appuyer ma recherche ${ }^{3}$. Il m'est arrivé aussi de m'impatienter parce que tardaient à venir les thèses et les monographies de collègues qui restaient sur le rivage pour débattre d'historiographie.

De telles préoccupations historiographiques sont compréhensibles. Tous ceux qui oeuvrent dans le domaine de l'histoire du mouvement ouvrier - et plus que quiconque, ceux qui y sont entrés par la porte de côté - reconnaissent la justesse de la réflexion d'Éric Hobsbawm, suivant laquelle les chercheurs en sciences humaines doivent se montrer aussi empressés à transformer le monde qu'à l'interpréter ${ }^{4}$. La consigne ne vaut pas seulement pour les chercheurs révolutionnaires. Au Canada, l'étude de l'histoire du mouvement ouvrier a plus souvent été un moyen qu'une fin en soi, du moins dans sa version anglaise.

À la bibliothèque du nouveau ministère du Travail, au début du siècle, l'histoire a été conçue comme un moyen de définir les institutions et de résoudre les conflits. La tentative faite par Mackenzie King afin de convaincre Adam Shortt de devenir un John Commons canadien, comme l'a rapporté Jay Atherton, n'a été qu'un échec partiel ${ }^{5}$.

\footnotetext{
${ }^{2} \quad \grave{A}$ titre d'exemple, voir S.B. Ryerson, «À propos de Les Syndicats Nationaux de Jacques Rouillard», Revue d' histoire de l'Amérique française, 35,3 (décembre 1981): 397-406.

3 H.B. Stowe, Uncle Tom's Cabin (éditions Collier, New York, 1962), 117. Il s'agit du livre Working People: An Illustrated History of Canadian Labour (Ottawa, 1980).

4 Éric Hobsbawn, «Labour History and Ideology», Journal of Social History, VII, 2 (été 1974); 380-381; cité par G.S. Kealey et P.Warrian (ed.), Essays in Canadian Working Class History (Toronto, 1976), 12.

Jay Atherton, «The Department of Labour and Industrial Relations, 1900-1914» (Carleton, M.A., 1972), 99. Pour une perspective historique générale, voir G.S. Kealey, «Looking Backward: Reflections on the Study of Class in Canada", History and Social Science Teacher, XVI, 4 (été 1981): 213-222.
} 
Au fil des années, certaines universités canadiennes en vinrent à accorder aux relations industrielles une place dans leur programme, bien qu'en appendice aux sciences économiques et politiques. Mais de modestes connaissances sur les syndicats et leurs fonctions finirent par faire partie du bagage des connaissances à acquérir. C'est le professeur Harold Logan qui, dans sa série d'ouvrages, tellement détaillés qu'ils lassent souvent le lecteur, a le mieux comblé le besoin ${ }^{6}$. Le champ d'étude s'est graduellement élargi, mais l'accent a toujours été mis sur les structures syndicales, les conflits ouvriers et des cas de négociations collectives qui ont tourné au vinaigre. À titre d'exemple, citons l'ouvrage de G.F. MacDowell, The Brandon Packers Strike?

Avec un engouement tout à fait compréhensible pour la politique, un courant quelque peu plus académique au sein de l'histoire du mouvement ouvrier est apparu au Canada anglais. Alors qu'il effectuait un travail de pionnier pour contester le mythe persistant qui voulait que sir John A. Macdonald ait été l'ami des travailleurs, Bernard Ostry ne s'est même pas donné la peine de lire le célèbre Trade Unions Act de 1872, où il aurait découvert qu'il s'agissait littéralement d'une fumisterie ${ }^{8}$. La première étude sérieuse sur la grève générale de Winnipeg, traditionnellement la plaque tournante de nos histoires classiques du mouvement ouvrier a été réalisée dans le cadre d'un vaste projet de recherche sur les débuts du Crédit social en Alberta ${ }^{9}$. Pour sa part, Kenneth McNaught, dans sa biographie de J. S. Woodsworth, a projeté un éclairage en évoquant le rôle des «labour churches», des dockers militants, et en décrivant le grand rêve de Woodsworth de former un véritable parti ouvrier ${ }^{10}$. Puis, en 1961, l'apparition du Nouveau parti démocratique, rejeton commun de l'ancien CCF et du nouveau Congrès du travail du Canada, a servi d'inspiration à plusieurs études sur la participation du mouvement syndical à la politique. Mentionnons notamment le tour d'horizon qu'a fait Martin Robin des innombrables organisations et fortes personnalités des premiers temps du militantisme du mouvement ouvrier, surtout dans l'Ouest, et la tentative ingénieuse de

\footnotetext{
6 H.A. Logan, Trade Unions in Canada (Toronto, 1948). Voir également State Intervention and Assistance in Collective Bargaining: The Canadian Experience, 1943-1954 (Toronto, 1956).

7 G.F. MacDowell, The Brandon Packers Strike: A Tragedy of Errors (Toronto, 1971). Voir également Stuart Jamieson, Times of Trouble: Labour Unrest and Industrial Conflict in Canada, 1900-1966 (Ottawa, 1966); John Crispo, International Unions in Canada (Toronto, 1967), deux livres influents d'envergure nationale.

8 Bernard Ostry, «Conservatives, Liberals and Labour in the 1870's», Canadian Historical Review, XLI, 2 (juin 1960); "Conservatives, Liberals and Labour in the 1880's», Canadian Journal of Economics and Political Science, XXVII, 2 (mai 1961), où l'auteur réfute le point de vue de Donald Creighton, «George Brown, Sir John A. Macdonald and the Working Man», Canadian Historical Review, XXIV, 4 (décembre 1943). (C'est le sénateur E.A. Forsey qui m'a servi de guide dans la démarche qui m'a amené à découvrir la maigre pertinence du Trade Unions Act, 1872).

D.C. Masters, The Winnipeg General Strike (Toronto, 1950).

1959).
} 
Gad Horowitz d'appliquer la théorie hartzienne à l'existence d'au moins un embryon de courant socialiste au Canada ${ }^{11}$.

Un troisième courant, beaucoup moins académique celui-là, mais plus influent en l'absence d'autres études, a tenté de répondre à une demande soutenue pour des histoires institutionnelles. Les syndicats et même les ministères du travail ont besoin de publications qui conviennent, dans le style et dans le ton, aux syndiqués, aux étudiants et au public qui s'intéressent aux questions syndicales. Comme de telles publications laissent une marque sur des esprits en voie de formation, elles mériteraient beaucoup plus de sérieux qu'elles n'en ont eu jusqu'à maintenant. Les histoires du mouvement ouvrier faites par Morden Lazarus, Jack Williams et Ed Seymour s'appuient sur les études de personnes comme Margaret Mackintosh, Leslie Wismer, Andy Andras et Howard Conquergood, ainsi que de journalistes dont les écrits ne sont pas répertoriés ${ }^{12}$. L'on pourrait classer dans une catégorie voisine des histoires commanditées, tels le livre de Paul Phillips sur la Fédération du travail de la Colombie-Britannique, No Power Greater, ou l'ouvrage de Warren Caragata, Alberta Labour, une histoire provinciale particulièrement bien faite en ce qui a trait aux premières années du mouvement ouvrier ${ }^{13}$.

Aucun projet d'histoire ouvrière commandité n'est resté aussi longtemps en préparation et n'a été aussi attendu que le livre de M. Eugene Forsey, Canadian Trade Unions, 1812-1902. Lancé par le Congrès du travail du Canada comme son projet du centenaire pour l'année 1967, l'ouvrage a été comprimé et a subi les coupures d'au moins trois maisons d'édition. Finalement, le livre est sorti des presses cette année, en bonne partie grâce aux pressions du Committee on Canadian Labour History (CCLH) et de quelques historiens du mouvement ouvrier. Comme l'ex-sénateur Forsey l'avait prédit, il sera l'objet de bien des critiques. En histoire du mouvement ouvrier, les modes ont changé depuis que cet ouvrage a été mis en chantier. Couronnement de bien des réalisations, cette étude présente une vue d'ensemble du

11 Martin Robin, Radical Politics and Canadian Labour, 1880-1930 (Kingston, 1968); Gad Horowitz, Canadian Labour in Politics (Toronto, 1978). Sur des thèmes connexes, voir Walter Young, The Anatomy of a Party: The National C.C.F., 1932-1961 (Toronto, 1969); William Rodney, Soldiers of the International: A History of the Communist Party of Canada, 1919-1929 (Toronto, 1968) et, un ouvrage rare sur un thème plus moderne: David Kwavnick, Organized Labour and Pressure Group Politics: The Canadian Labour Congress, 1956-1968 (Montreal, 1972).

12 Morden Lazarus, Years of Hard Labour (Don Mills, 1974) et Up From the Ranks: Trade Union VIPs Past and Present (Toronto, 1977); Ed Seymour, An Illustrated History of Canadian Labour, 1800-1974 (Ottawa, 1976); Jack Williams, The Story of Unions in Canada (Toronto, 1975); E.A. Forsey, «History of the Labour Movement in Canada», Canadian Year Book, 1967 (Ottawa, 1967); C.A. Scotton, A Brief History of Canadian Labour (Ottawa, 1956); A.M. Kruger, R.O. MacDowell et L.A. MacDowell, Trade Unions and Collective Bargaining in Canada (Don Mills, n.d.).

13 Paul Philips, No Power Greater: A Century of Labour in B.C. (Vancouver, 1967); Warren Caragata, Alberta Labour: A Heritage Untold (Toronto, 1979). 
syndicalisme au Canada après la Confédération; elle constitue également un inventaire minitieux de la pensée et des aspirations de cette brave minorité d'hommes et de femmes qui ont consacré une partie de leur vie au syndicalisme. C'est également grâce à l'esprit visionnaire de Forsey qu'une équipe de chercheurs s'est attaquée à la tâche de sauvegarder les archives syndicales à travers le pays au début des années soixante. La valeur de ce qu'ils ont récupéré est mise en relief par ce qu'ils n'ont pas réussi à sauver, souvent par un cheveu ${ }^{14}$. Ceux qui sont en désaccord avec Forsey devraient éviter de succomber au péché mignon du critique paresseux et soutenir que l'auteur aurait dû écrire un autre ouvrage que celui qu'il a fait.

Mais toutes les histoires du mouvement ouvrier ne se sont pas confinées à l'étude de l'organisation syndicale, des grèves et de la politique. Quelques rares études, dont celle de Doris French, Faith, Sweat and Politics, biographie de Daniel O'Donoghue, ont tenté de tirer de l'obscurité les héros de la classe ouvrière. Comme exemples typiques de ce genre, citons les panégyriques produits par Progress Books ${ }^{15}$.Dans le monde académique, quelques articles sérieux ont ouvert la voie à un genre différent: l'article de John Irwin Cooper sur les débardeurs de Québec, l'étude de H. Clare Pentland sur les grèves du canal Lachine dans les années 1840, et l'analyse faite par Frank W. Watt de la presse ouvrière dans une Ontario en voie d'industrialisation ${ }^{16}$.

Tout cela constituait une assise bien fragile pour soutenir le «baby boom» des universités, dont les finissants puisaient leur inspiration historique dans le militantisme des années soixante et qui recherchaient beaucoup mieux que ces vieux thèmes éculés qu'étaient la politique, la religion et les relations extérieures. Le résultat fut assez impressionnant. Des 276 mémoires ou thèses de maîtrise et de doctorat inscrites à la Société historique du Canada en 1966, quatre seulement prétendaient traiter de thèmes se rapportant à l'histoire du mouvement ouvrier et deux seulement appartiennent à l'histoire du mouvement ouvrier comme telle. Dix ans plus tard, en 1976, le nombre de thèses inscrites est de

14 E.A. Forsey, Trade Unions in Canada, 1812-1900 (Toronto \& Buffalo, 1982). Voir Nancy Stunden, «Labour, Record and Archives: The Struggle for a Heritage», Archivaria, 4 (1977): 74-75.

is Doris French, Faith, Sweat and Politics: The Early Union Years in Canada (Toronto, 1962). Sur le style de Progress Books, voir par exemple, Catherine Vance, Not by Gods but by People: The Story of Bella Hall Gauld (Toronto, 1968); Louise Watson, She Was Never Afraid: The Biography of Annie Buller (Toronto, 1976); Tom McEwen, The Forge Glows Red: From Blacksmith to Revolutionary (Toronto, 1974); Oscar Ryan, Tim Buck: A Conscience for Canada (Toronto, 1975) que l'on pourrait comparer à l'ouvrage un peu moins guindé de Phillis Clarke et William Beeching (ed.): Yours in the Struggle: Reminiscences of Tim Buck (Toronto, 1977). L'expression est de Kealey et Hann (ed.), Essays in Working Class History, 188.

16 J.I. Cooper, "The Quebec Ship-Labourers' Benevolent Society», Canadian Historical Review, XXX, 4 (décembre 1949); H.C. Pentland, "The Lachine Strike of 1843», ibid., XXIX, 3 (septembre 1948); F.W. Watt, «Literature of Protest» in Carl Klinck, Literary History of Canada (Toronto, 1965) et «The National Policy, the Workingman and the Proletarian Ideas in Victorian Canada», Canadian Historical Review, XL, 1 (mars 1959). 
1 077. Parmi elles, 277 portent sur l'histoire sociale, et 52, dont 11 en français, traitent de thèmes se rapportant au mouvement ouvrier ${ }^{17}$.

Comme dans tout autre processus de changement historique, cette transformation a été aussi bien qualitative que quantitative, bien qu'elle ait également revêtu un caractère provisoire. Tout comme chez leurs maîtres, les premiers parmi ces nouveaux historiens, indépendamment de leurs orientations politiques, vouaient d'abord allégeance à la science historique. Ils recueillaient les données telles qu'ils les trouvaient et ils fouillaient pour en trouver d'autres, choisissant des sujets qui se prêtaient à un examen approfondi. Il est fort possible qu'Irving Abella ait été trop captivé par le sympathique J.B. Salsberg lorsqu'il a repris l'interprétation communiste au sujet de l'implantation des syndicats industriels au Canada - Charles Millard aurait certainement proposé une interprétation quelque peu différente - néanmoins, Abella se faisait un devoir d'être fidèle aux sources ${ }^{18}$.

Les historiens dont les travaux ont commencé à paraître au début des années soixante-dix se sont établi une réputation enviable. La plupart d'entre eux se sont concentrés sur le début du $\mathrm{XX}^{\mathrm{e}}$ siècle. Personne ne s'est rendu plus loin qu'Abella, dont les travaux se sont terminés à la veille de la fusion du Congrès des Métiers et du Travail du Canada et du Congrès Canadien du Travail. Terry Copp a commencé ses recherches sur la pauvreté à Montréal à partir de l'étude de «la ville en bas de la côte» de Herbert Ames, mais sa recherche persistante de données lui a permis d'établir toutes les dimensions de la vie institutionnelle de la classe ouvrière. Dans son ouvrage Dangerous Foreigners, Donald Avery s'est indigné de la froideur des archives sur l'immigration et l'exploitation ouvrière ainsi que sur la réplique militante que cette dernière a suscitée, mais il ne s'en est pas moins cantonné aux sources traditionnelles. Pour sa part, Ross McCormack, dans Reformers, Rebels and Revolutionaries, a repris de façon beaucoup plus précise et systématique ce que Martin Robin avait amorcé presque dix ans plus tôt, cette fois en restreignant son objet d'étude à l'Ouest canadien et en se limitant à reconstituer l'enchaînement des courants qui se sont conjugués à Winnipeg en $1919^{19}$.

C'est David Bercuson, le plus important et le plus influent des jeunes historiens du mouvement ouvrier appartenant à une école métho-

17 Canadian Historical Association, Register of Dissertations, I (1966); XI (1976).

18 Irving Abella, Nationalism, Communism and Canadian Labour (Toronto, 1973).

19 J.T. Copp, The Anatomy of Poverty: The Condition of the Working Class in Montreal, 1897-1929 (Toronto, 1974). (Voir également H.B. Ames, The City Below the Hill (Toronto, 1972), l'une des très précieuses séries de réimpressions de classiques anciens ou clandestins publiées par la University of Toronto Press à une époque plus prospère.) Donald Avery, ‘Dangerous Foreigners $>$ : European Immigrant Workers and Labour Radicalism in Canada, 1896-1932 (Toronto, 1979); Ross McCormack, Reformers, Rebels and Revolutionaries: The Western Canadian Radical Movement, $1899-1919$ (Toronto, 1978). 
dologique quelque peu traditionnelle, qui a su réaliser l'étude la plus globale et la plus innovatrice. Dans son ouvrage Confrontation at Winnipeg, il a effectué une étude détaillée des structures sociales et commerciales de la ville entière. Cela lui a permis de mettre en lumière le fait que les anciens prolétaires qui avaient réussi par eux-mêmes à se hisser au sommet de la structure sociale étaient parmi les moins enclins à faire une place au syndicalisme. La droite avait toujours craint la grève générale; la gauche avait toujours souhaité son avènement. Bercuson a pris les grévistes au mot: ils s'étaient engagés dans une rude épreuve de négociation collective et ils connurent une défaite amère ${ }^{20}$. Dans ce qui constitue à toute fin pratique la suite de ses recherches sur la grève générale de Winnipeg, Bercuson fait ressortir la même idée. Dès le départ, l'expansion de la One Big Union (OBU) a été entravée par les contradictions et la confusion de ses fondateurs, mais l'échec de cette centrale s'explique surtout par le fait qu'elle n'a pas réussi à améliorer le sort matériel des syndiqués. Il s'agit là d'une leçon que les syndicalistes communistes, entre autres, n'ont pas tardé à tirer ${ }^{21}$.

Quelque temps plus tard, dans un essai de catégorisation, Bryan Palmer a tenté de faire une distinction entre les historiens canadiens du mouvement ouvrier de la «première génération» et ceux de la «deuxième génération ${ }^{22}$. Comme toutes les constructions humaines, son étude vaut ce qu'elle vaut. Éparpillés géographiquement et isolés par leurs inhibitions à l'égard de la théorie, les historiens canadiens-anglais ont tendance à demeurer de sympathiques individualistes. Certes il y a eu des écoles d'interprétation, mais elles ont généralement été composées uniquement du maître - un Creighton, un W.L. Morton - et de quelques disciples errants et peu fiables qu'on peut toujours, le cas échéant, réunir pour un festschrift. Les historiens du mouvement ouvrier ont à peine réussi à trouver suffisamment en commun pour mettre sur pied un comité rattaché à la Société historique du Canada ${ }^{23}$.

Que cette «première génération» d'historiens ait existé ou non, la présence de la seconde ne fait aucun doute. Il est possible de la qualifier

20 David J. Bercuson, Confrontation at Winnipeg: Labour, Industrial Relations and the General Strike (Montréal, 1974). Sur les suites voir J.E. Rea, «The Politics of Conscience: Winnipeg After the Strike», Canadian Historical Association, Historical Papers, 1971. Winnipeg sert également d'objet d'étude à l'une des meilleures nouvelles histoires urbaines. Cette histoire urbaine pourrait éventuellement servir comme une des études de base pour écrire une histoire d'ensemble de la classe ouvrière: Alan J. Artibise, Winnipeg: A Social History of Urban Growth, 1874-1914 (Montreal and London, 1978).

21 David J. Bercuson, Fools and Wise Men: The Rise and Fall of the One Big Union (Toronto, 1978). (Le titre puise son inspiration d'une affirmation du pionnier de l'OBU, W.A. Pritchard: «Seuls les imbéciles essaient de faire des révolutions; les personnes sages s'y conforment».

22 Bryan D. Palmer, «Working-Class Canada: Recent Historical Writing», Queen's Quarterly, LXXXVI, 4 (hiver 1979-80): 599-601

${ }_{23} \mathrm{Au}$ Canada anglais, la profession a été décrite de façon habile par Carl Berger, The Writing of Canadian History: Aspects of English-Canadian Historical Writing, 1900 to 1970 (Toronto, 1976), surtout dans la conclusion. 
de diverses façons, mais aucune catégorie ne parvient à englober tous les attributs de cette «deuxième génération». Certaines de ses têtes de file, tels Peter Warian et Greg Kealey, comptaient parmi les leaders du mouvement étudiant de la fin des années 1960, au moment où celui-ci traversait sa phase finale, la plus radicale. Leur expérience dans le mouvement étudiant leur a laissé des souvenirs d'affrontements acerbes avec les sociaux-démocrates sur les campus universitaires et même au sein du Nouveau Parti Démocratique (NPD) ${ }^{24}$.

Mais peut-être est-ce encore la véritable distinction entre générations qui justifie le mieux le concept de deux générations différentes. S'il était facile de poursuivre des études avancées sur le mouvement ouvrier, on ne peut en dire autant des débouchés sur le marché du travail. Pendant une certaine période, du début des années '60 jusqu'au début des années '70, plusieurs postes s'ouvrirent dans les universités anglophones, mais cette période fut de courte durée. Alors que la plupart des membres de la première génération avaient réussi à s'installer confortablement à l'intérieur du réseau universitaire, la seconde génération vivait l'insécurité d'en être tenue à l'écart. Ce qui aurait pu devenir une pure Wissenschaften devint forcément Gemeineschaften. Dans la course aux emplois, qu'il s'agisse de postes temporaires ou des quelques rares postes permanents, une approche idéologique commune servait d'appui mutuel. C'est ainsi que les conditions économiques et objectives ont engendré un réseau dont on connaît peu de précédents dans la vie académique au Canada ${ }^{25}$.

Mais sur le plan historiographique, il va sans dire que c'est l'approche historique commune qui a préséance sur le partage d'une expérience vécue. Le point de vue de la «deuxième génération» ressort clairement dans les introductions, avant-propos, notes critiques et longs articles bibliographiques, comme les pages d'introduction de Russell Hann au livre Primary Sources in Working Class History et le tour de force de Greg Kealey dans le numéro 7 de la revue Labour/Le Travailleur. Avec l'enthousiasme qui caractérise les néophytes, les principaux représentants du groupe ratent rarement l'occasion de condamner le caractère étroit et «réductionniste» des approches institutionnelle et

24 Les conflits bourdonnent dans des articles comme celui de Terry Morley, «Canada and the Romantic Left», Queen's Quarterly, LXXXVI, 1 (printemps 1979) et dans la réplique de Palmer, ibid., 606, et Kealey, «Labour and Working-Class», 68.

${ }_{25}$ Voir Palmer, «Working-Class Canada», 606. Kealey met en doute l'affirmation: «J'ai obtenu le premier emploi pour lequel j'ai postulé.» D'autres - Palmer, Roberts, Hann — ont éprouvé plus de difficultés, même s'ils ont tous tiré parti des pressions exercées pour attribuer certains des rares postes disponibles à des historiens radicaux spécialisés en histoire sociale. Voir Kealey à l'auteur, le 17 mai 1982. 
politique ainsi que les faiblesses particulières de leurs quasicontemporains $^{26}$.

Dans un style rappelant celui des manifestes révolutionnaires, les membres de la «deuxième génération» affirment qu'avant leur arrivée bien peu de choses valables ont été accomplies. Dans un passage souvent cité, tiré de l'introduction à Essays in Working Class History, Kealey et Warrian soutiennent avec vigueur que l'histoire du mouvement ouvrier a été «... une catégorie de l'économie politique, un problème de relations industrielles, une ode à des dirigeants de la classe ouvrière, une chronique de locaux syndicaux ou un récit de grèves militantes... ${ }^{27} \grave{\mathrm{A}}$ leurs dires, l'histoire canadienne dans son ensemble avait stagné dans les bas-fonds du «centralisme». De tous leurs vénérables aînés, seul Maurice Careless avec ses recherches sur les «limited identities au Canada» avait su offrir une orientation qui méritait d'être suivie $^{28}$.

Faute d'inspiration suffisante au pays, la nouvelle génération est allée faire des études supérieures à l'étranger. À l'Université Rochester, Kealey a suivi la trajectoire typique d'un Canadien qui doit aller chercher une reconnaissance à l'extérieur avant d'être reconnu au Canada, ayant gagné l'estime de son très respecté maître à penser, Herbert Gutman $^{29}$. Une quantité considérable d'archives syndicales canadiennes se retrouvant aux États-Unis, les universités américaines pouvaient devenir des lieux de choix où poursuivre des études. Même si des étudiants canadiens avaient peu à apprendre sur le Canada dans les universités américaines, britanniques ou françaises, ces dernières pouvaient leur permettre d'échapper à la sclérose de Toronto ou de Montréal. Sous la direction d'un Gutman, d'un Eugène Genovese, d'un Louis Chevalier ou d'un David Montgomery, ils avaient l'occasion de découvrir qu'on pouvait aborder l'histoire du mouvement ouvrier de façon très différente et d'une manière plus dynamique ${ }^{30}$.

Mais autant ces influences ont été importantes, autant le chefd'oeuvre de E.P. Thompson, The Making of the English Working Class, publié en 1963, a été le fons et origo de la «nouvelle» histoire du mou-

\footnotetext{
26 Russell Hann, «Introduction» in Russell Hann, Greg Kealey, Linda Kealey, Peter Warrian (ed.), Primary Sources in Canadian Working Class History (Kitchener, 1973); Kealey, «Labour and Working Class»; et G.S. Kealey, «H.C. Pentland and Working Class Studies», Canadian Journal of Political and Social Theory, III, 2 (1979): 79-94.

${ }_{27}$ Kealey \& Warrian, Essays, 8 (La traduction est de nous).

28 Kealey, «The Working Class», 116; Palmer, «Working-Class Canada», 598. Voir J.M.S Careless, «Limited Identities in Canada», Canadian Historical Review, L, 1 (mars 1969): 1-10 et également Stanley Mealing, «The Concept of Social Class in the Interpretation of Canadian History», ibid., XLVI, 3 (septembre 1964): 201-218.

29 McNaught, «Thompson vs. Logan», 144n. Voir H.G. Gutman et G.S. Kealey (ed.) Many Pasts, 2 vol., (Englewood Cliffs, N.J., 1973)

30 Hann et al., Primary Sources, 12 ss; B.D. Palmer, «Most Uncommon Common Men: Crafts and Culture in Historical Perspective», Labour/Le Travailleur, I (1976): 5-31.
} 
vement ouvrier. Aucun historien britannique n'avait exercé autant d'influence sur nos historiens canadiens depuis que l'évêque Stubbs et sir Frederick Maitland avaient forcé des générations d'historiens à accorder une importance exagérée aux constitutions - et pourtant le Canada n'est plus, même sur le plan formel, une colonie! L'influence de Thompson s'explique par des raisons tout à fait légitimes. Peu importe sa dette de reconnaissance envers Raymond Williams et l'école des Annales, Thompson a réussi à revigorer le marxisme comme outil d'analyse historique, laissant tomber en désuétude les vieux schémas mécanistes. Les classes sociales ne sont plus des constructions statiques mais des organismes. D'après Thompson, «si on arrête l'histoire à un point précis, dès lors, on ne peut plus parler de classes mais d'une multitude d'individus vivant une multitude d'expériences. Mais si on observe ces individus pendant une période de transformations sociales suffisamment longue, l'on peut dégager le profil de leurs relations, de leurs idées et de leurs institutions. Ce sont les hommes qui définissent les classes sociales en faisant l'expérience de leur propre histoire et, en dernière instance, il s'agit de la seule définition valable des classes sociales» ${ }^{31}$.

Des choses si sensées sont-elles vraiment si rares dans les cercles académiques qu'elles puissent enfanter des révolutions? Mais, question encore plus pertinente, le marxisme empirique de Thompson est-il applicable au contexte canadien? S'il existe chez cette «deuxième» génération d'historiens du mouvement ouvrier un point de convergence, il semble que ce soit un engagement à trouver réponse à cette question, qu'il s'agisse comme pour Palmer, Hann, Kealey ou Craig Heron d'une préoccupation centrale ou, pour d'autres, d'une préoccupation inconsciente. Si Kealey avait déjà exposé son point de vue en 1973 lors de la réimpression d'un travail étudiant et de sa publication commentée de la Commission royale d'enquête sur les relations entre le capital et le travail de 1889, il aura fallu attendre la publication de Primary Sources in Canadian Working Class History, subventionnée par un projet Perspectives-jeunesse, pour connaître la première prise de position d'importance du groupe ${ }^{32}$. L'on y trouve un inventaire de toute la gamme de matériel disponible dans les bibliothèques et archives canadiennes, permettant d'écrire l'histoire à partir du modèle de Thompson. Comme l'expliquait Kealey (en collaboration avec Russell Hann) aux lecteurs de Archivaria en 1977, il n'y avait pratiquement pas de limites à ce qu'on pouvait ajouter à la liste. Cette «nouvelle» façon d'écrire l'histoire nécessitait des dossiers judiciaires et de police,

31 E.P. Thompson, The Making of the English Working Class (London, 1963). Sur les débats en Grande-Bretagne et ailleurs, voir Kealey, "Labour and Working Class», 80-91. Voir également Raymond Williams, Culture and Society (London, 1959). (La traduction est de nous).

32 Hann et al., Primary Sources, 12-13. Voir également G.S. Kealey (ed.), Canada Investigates Industrialism: The Royal Commission on the Relations of Labour and Capital, 1889 (Toronto, 1973), xxvii; G.S. Kealey, Working Class Toronto at the Turn of the Century (Toronto, 1973). 
des documents d'affaires, des listes de paie, des procès-verbaux des assemblées de loges orangistes et de confréries ou sociétés de secours mutuels, ainsi que la presse ouvrière radicale aussi bien que populaire et même le discours idiomatique, soigneusement recueilli par J.W. Bengough dans Grip ${ }^{33}$.

En tant que groupe relativement cohérent, la deuxième génération d'historiens s'est sentie en mesure de planifier son travail. Kealey et Palmer se sont engagés à produire une nouvelle synthèse de l'histoire du Canada qui tiendrait davantage compte de la lutte et des conflits de classe. Il n'en demeure pas moins que leurs priorités immédiates sont restées le reflet de la décennie pendant laquelle ils ont été les plus prolifiques. Dans un Canada qui se décentralise de plus en plus rapidement, ils ont centré leur attention sur les régions et même sur certaines communautés. En étudiant de larges tranches de l'histoire de Hamilton comme Palmer et Heron ont commencé à le faire, et en effectuant des recherches sur l'histoire d'un Toronto en voie d'industrialisation entre 1860 et 1892 comme celles que Kealey a menées, l'on peut espérer pouvoir dégager des «patterns» de culture, de conflits et de contrôle sur les lieux de travail ${ }^{34}$. Étant donné l'absence d'une gamme étendue et riche de sources secondaires comme celles auxquelles Thompson a eu accès, et le caractère hétérogène des populations, des conditions économiques et des étapes de développement propres au Canada, la rédaction de monographies s'imposait dans un premier temps.

En tant qu'entreprise historique, les travaux des nouveaux historiens du mouvement ouvrier ont suscité passablement d'admiration et de respect. Le fait que le prix Macdonald ait été décerné à l'oeuvre Toronto Workers de Greg Kealey a largement été accueilli non seulement comme une reconnaissance de la valeur de l'ouvrage lui-même, mais également comme un hommage à son auteur pour son rôle prodigieux comme éditeur, auteur et pasteur du troupeau. De plus, il faut reconnaître que le groupe n'a jamais manifesté d'intransigeance et d'étroitesse d'esprit, et je suis, pour ma part, bien placé pour en témoigner. Au début des années '70, Michael Cross, un historien plus âgé et alors plus connu, se découvrit un enthousiasme qu'il transporta avec lui de Toronto à l'Université Dalhousie, mettant ainsi en place les assises académiques qui permettraient à Kealey, Heron, David Frank, Ian McKay et à la revue Labour/Le Travailleur de fonctionner pendant le reste de la décennie. L'essai bibliographique dans lequel Cross adopte

33 G.S. Kealey, «Documenting Working Class History: North American Traditions and New Approaches», Archivaria, 4 (1977): 100-114 (par contraste ironique avec l'article de Stunden qui met l'accent presque exclusivement sur la cueillette et la conservation d'archives et de documents syndicaux).

34 Hann et al., Primary Sources, 13-14; B.D. Palmer, A Culture in Conflict: Skilled Workers and Industrial Capitalism in Hamilton, Ontario, 1860-1914 (Montreal, 1979); Craig Heron, «The Working Class in Hamilton, 1890-1930» (Dalhousie, Ph.D. 1981); G.S. Kealey, Toronto Workers Respond to Industrial Capitalism, 1867-1892 (Toronto, 1980). 
un ton railleur à l'égard de lui-même, publié dans la revue Labour/Le Travailleur, constituait une contribution majeure au premier numéro de cette revue $^{35}$.

Une telle concentration de ressources, soutenues par des subventions fédérales, a contribué à orienter des chercheurs, autrefois trop peu nombreux, vers l'histoire du mouvement ouvrier dans les provinces Maritimes, reléguant pour une fois dans l'ombre l'Ouest canadien déjà trop connu. Alors qu'à une certaine époque, la région canadienne où le mouvement ouvrier a connu l'histoire la plus traumatisante ne disposait que de l'histoire de l'autodidacte Paul MacEwan, d'importantes recherches sont présentement en cours ${ }^{36}$. Avant d'avoir subi, du moins autant qu'on puisse en juger, l'influence des nouveaux venus, l'Université Dalhousie avait déjà produit la meilleure étude d'histoire sociale de la décennie, The Winter's Tale de Judith Fingard, publiée en 1974. Même si le sujet, par définition, se limite à l'influence de l'hiver sur la vie des Canadiens de l'époque pré-industrielle, l'étude n'en fourmille pas moins de références importantes à presque toutes les époques de notre passé $e^{37}$.

Il va sans dire que l'accueil très favorable réservé à l'oeuvre des historiens de la «deuxième génération» n'a pas été unanime. Certains ont eu une réaction tout à fait légitime à des critiques de caractère historiographique. Alors qu'il existe chez les historiens canadiensanglais une tradition bien établie de critiquer la façon dont le travail a été accompli, la remise en question de la nature du travail réalisé et de sa justification, est restée peu développée. Il y a peut-être trop longtemps que cette critique se fait attendre. Les historiens qui concentrent leurs recherches sur les syndicats, les conflits de travail ou le rôle des dirigeants syndicaux, devront dorénavant, comme Kenneth McNaught l'a fait récemment, fournir des arguments pour étayer leurs thèses. Ils devront fournir la preuve que les dirigeants syndicaux ont joué un rôle fondamental quant au sort des ouvriers et que les syndicats revêtent autant d'importance que les loges orangistes, les «charivaris» ou les «whitecapping» de circonstance. Ce sont souvent ces formes primaires d'associations qui ont donné naissance aux luttes syndicales ${ }^{38}$.

L'un des reproches les plus fréquemment adressés aux historiens de la «deuxième génération», c'est qu'ils se sont dépensés davantage à

35 Voir M.S. Cross, «To the Dartmouth Station: a worker's-eye view of Labour History», Labour/Le Travailleur, I (1976): 193-208. Voir également «The Shiners' War: Social Violence in the Ottawa Valley in the 1930's», Canadian Historical Review, LIV, 1 (mars 1973).

36 Paul McEwan, Miners and Steelworkers: Labour in Cape Breton (Toronto, 1976) (dont une partie avait déjà paru dans The Highlander). Voir David Frank, «Class Conflict in the Coal Industry, Cape Breton, 1922» in Kealey \& Warrian, Essays, 161-184.

Judith Fingard, «The Winter's Tale: Contours of Pre-Industrial Poverty in British America, 1815-1860», Canadian Historical Association, Historical Papers, 1974, 65-94. Pour un autre exemple de ses recherches voir Jack in Port: Sailor towns of Eastern Canada (Toronto, 1982).

38 McNaught, «Thompson vs Logan», 167-168. Voir H.C. Pentland, «The Canadian Industrial Relations System: Some Formative Factors», Labour/Le Travailleur, IV (1979): surtout 23. 
établir le sens de cette «nouvelle» histoire et à discourir sur la contribution de E.P. Thompson, qu'à en démontrer la portée pratique dans le contexte canadien. Compte tenu de ce qu'on nous avait promis, le bilan suivant nous semble un peu mince: publication de deux thèses, un livre sur les Chevaliers du travail, et publication d'articles et de quelques chapitres de thèses éparpillés dans des revues scientifiques. Bien plus, la «nouvelle» histoire ne revêt pas toujours le caractère innovateur auquel on était en droit de s'attendre. Malgré la qualité de plusieurs études, les travaux qui s'inscrivent dans le sillage du manifeste de Kealey et de Warrian se confinent aux mêmes limites - syndicats, leaders et grèves - que les auteurs de ces travaux semblent déplorer chez les historiens traditionnels. L'oeuvre de Palmer, A culture in Conflict, passe d'une analyse de la culture ouvrière à Hamilton, qui semble davantage reposer sur l'idéologie que sur une base documentaire, à un relevé utile mais traditionnel des syndicats, des conflits de travail et de la politique ouvrière de la ville. Même le livre Toronto Workers, de loin supérieur à celui de Palmer, est condamné par ses sources à ne pas trop s'éloigner du terrain de la politique, des leaders et des organisations. Comme le fait remarquer David Bercuson, un bon tiers du livre de Kealey est consacré à faire le récit vivant de la politique torontoise dans les années $1880^{39}$.

Mais ce sont davantage les prétentions exagérées des historiens de la «deuxième génération» qui engendrent de telles critiques, qu'un bilan réaliste de ce qu'ils sont tentés d'accomplir. En guise de tu quoque, l'on pourrait ajouter que Thompson consacre pratiquement la moitié de son oeuvre The Making of the English Working Class à réécrire l'histoire de la politique radicale anglaise. Palmer, et encore bien plus Kealey, ont abordé les contradictions et la complexité de la politique et de la culture ouvrières avec une honnêteté qui a dû perturber leurs collègues plus pudiques. En effet, pour tout Canadien anglais «progressiste» et fidèle à lui-même, il n'est pas facile de fraterniser avec les loges orangistes, même de loin. Pourtant pour écrire l'histoire du $19^{\mathrm{e}}$ siècle de façon honnête, il faut nécessairement tenir compte des préjugés religieux, des problèmes aigüs d'alcoolisme, de la violence familiale et du racisme ${ }^{40}$.

Certaines critiques plus justifiées, encore une fois formulées surtout par Bercuson, remettent en question la pertinence dans le contexte canadien du concept de culture ouvrière mis au point par Thompson.

39 D.J. Bercuson, «Through the Looking Glass of Culture: An Essay on the New Labour History and Working Class Culture in Recent Canadian Historical Writing», ibid., VII (printemps 1981): 95 ff.; McNaught, «Thompson vs Logan», 150-151. Dans la version publiée aux éditions Open University, sur un total de 939 p., l'auteur consacre 424 p. à l'histoire politique.

40 Par exemple, G.S. Kealey, «The Orange Order in Toronto: Religion, Riot and the Working Class», Kealey \& Warrian, Essays, 13-34 (paraît également comme le chap. VII de Toronto Workers); B.D. Palmer, «Discordant Noises: Charivaris and Whitecapping in Nineteenth Century North America», Labour/le Travailleur, III (1978): 5-62 et chap. I-II de A Culture in Conflict. 
Ce qui s'applique à la soi-disant homogénéité du nord de l'Angleterre au $18^{\mathrm{e}}$ siècle ne convient pas nécessairement au Canada. Le Canada est, comme dans le passé, un pays différent, où vivent des personnes différentes. Pour s'en rendre compte, l'on n'a qu'à faire un survol de l'histoire de la classe ouvrière. Cette histoire doit tenir compte des différences régionales, ethniques, religieuses et linguistiques, dans un ordre politique qui a sciemment mis en relief ces clivages, possiblement comme antidote à la solidarité de classe. Bercuson réfute assez rapidement les prétentions de Palmer selon lesquelles «une vie communautaire intense» aurait existé chez les travailleurs de Hamilton. Kealey serait trop sensé pour faire pareille affirmation au sujet de Toronto, même s'il s'est engagé dans une démarche laborieuse, et non sans un certain succès, qui visait à atténuer l'importance des conflits partisans et religieux qui ont mérité à la Ville-reine son titre de «Belfast de l'Amérique du Nord» ${ }^{41}$.

Au sein même de la classe ouvrière, la place des artisans spécialisés a longtemps été l'objet d'équivoque. Rejetant de façon passionnée l'épithète de «petits bourgeois», Palmer soutient que les artisans ne sont rien de moins que le «fer de lance du mouvement ouvrier», livrant une lutte d'arrière-garde pour conserver le contrôle des lieux de travail. D’une manière caractéristique, Kealey, plus nuancé, perçoit ces derniers comme étant à la fois tournés vers le passé et orientés vers l'avenir ${ }^{42}$. Mais quelle que soit leur orientation, comme Roland et ses chevaliers, ils sont voués à la défaite, de la même façon que les mouleurs, les monteurs de poêles et les polisseurs de l'établissement Gurney qui, victimes de leurs structures syndicales fondées sur leurs métiers respectifs, ont été les perdants ${ }^{43}$.

Paradoxalement, tout en subissant la défaite sur les lieux de travail, les syndicats de métiers survivront comme forme dominante du syndicalisme en Amérique du Nord pendant deux autres générations. Y a-t-il une distinction fondamentale à faire entre ces artisans, «fer de lance du mouvement ouvrier», et les syndicalistes d'affaires suffisants de la Fédération Américaine du Travail et du Congrès des Métiers et du Travail du Canada? Les imprimeurs syndicalisés qui font des compromis avec la compagnie Mergenthaler dans le but de prendre le contrôle de la linotypie battent-ils en retraite ou livrent-ils un combat courageux contre le destin ${ }^{44}$ ? Et mettant l'accent sur la scène locale et sur des cas précis, les «nouveaux» historiens ne courent-ils pas le risque de laisser

\footnotetext{
41 Bercuson, «Through the Looking Class», 96-110.

42 Par exemple, Palmer, Culture in Conflict, xii; Kealey, «Labour and working Class», 98. Voir Palmer, «Uncommon Common Men», supra.

${ }_{43}$ Craig Heron and Bryan Palmer, «Through the Prism of the Strike; Industrial Conflict in Southern Ontario, 1901-1914», Canadian Historical Review, LVIII, 4 (décembre 1977).

44 Voir, par exemple, Wayne Roberts, «The Last Artisans: Toronto Printers, 1896-1914», Kealey and Warrian, Essays, 125-142; Elizabeth Baker, Printers and Technology (New York, 1957).
} 
dans l'ombre ce qui se passe dans un monde ouvrier sous l'influence de Sam Gompers et du congrès de Berlin de 1902? À quel moment les nobles artisans sont-ils devenus les égoïstes aristocrates ouvriers? Selon Paul Craven, «notre sympathie pour l'artisan fier de jadis... ne doit pas nous faire perdre de vue que les syndicats de métiers du début du XX $\mathrm{X}^{\mathrm{e}}$ siècle... n'étaient plus au diapason des préoccupations de la classe industrielle dans son ensemble» ${ }^{45}$.

Bien entendu, du point de vue des approches de l'histoire du mouvement ouvrier au Canada anglais, Craven se situe dans un courant plutôt différent, bien qu'aussi «marxisant». Il s'agit d'une école qui s'est inspirée de l'histoire nationaliste d'un Donald Creighton ou de l'économie politique nationaliste de Harold Innis. En matière d'histoire du mouvement ouvrier, à défaut d'y trouver de la précision, cette école pouvait toujours trouver du réconfort dans l'oeuvre de Charles Lipton, Histoire du syndicalisme au Canada et au Québec ${ }^{46}$, qui est la première à suivre l'inspiration du nationalisme marxiste. Robert Laxer a apprêté le même thème à une sauce plus moderne dans son livre Canada's Unions $^{47}$. Le nationalisme ouvrier n'a pas trouvé d'écho très favorable auprès des historiens de la «deuxième génération». Pour Russell Hann, ce courant n'est que du «néo-creightonisme», un intérêt historique pour les travailleurs qui s'épanouit... seulement quand ces derniers se vendent aux unions internationales américaines ou se séparent d'une union internationale pour créer un syndicat canadien indépendant ${ }^{48}$.

Au moins un livre, Gompers in Canada de Robert Babcock, semblait, du moins après un examen superficiel, se mériter cette accusation ainsi que d'autres reproches bien connus. Dans cet ouvrage, l'auteur attribue carrément la victoire des syndicats internationaux au sein du CMTC à l'impérialisme de l'AFL. Il s'agissait non seulement d'un thème nationaliste (bien qu'écrit par un Américain) mais d'une approche qui restait tellement indifférente aux points de vue des militants à la base qu'elle a ignoré les motivations particulières et les affiliations des participants au congrès de Berlin ${ }^{49}$. Un livre plus récent, celui de Sally Zerker portant sur l'histoire de la Toronto Typographical Union, perd l'histoire du plus ancien syndicat permanent au Canada dans un mélodrame similaire d'immixtion et de vexations américaines ${ }^{50}$.

L'école de l'économie politique ne conduit pas nécessairement à se préoccuper de la question de l'indépendance du Canada. Dans une

45 Paul Craven, ‘An Impartial Umpire `: Industrial Relations and the Canadian State, 19001911 (Toronto \& Buffalo, 1980), 139.

46 Charles Lipton, The Trade Union Movement of Canada, 1827-1959 (Toronto, 1973, édition revue et corrigée).

47 Robert Laxer, Canada's Unions (Toronto, 1976).

48 Hann et al., Primary Sources, 11.

49 Robert Babcock, Gompers in Canada: A Study in American Continentalism Before the First World War (Toronto \& Buffalo, 1975).

50 Sally Zerker, The Rise and Fall of the Toronto Typographical Union, 1832-1972: A Case Study in Foreign Domination (Toronto \& Buffalo, 1982). 
étude qui constitue le pendant de la perspective de Copp, Michael Piva a réalisé une précieuse série d'études statistiques et institutionnelles regroupées dans son ouvrage The Condition of the Working Class in Toronto $^{51}$. En tant que critique implicite et explicite de la «nouvelle» histoire, Piva soutient qu'il est prématuré et peu opportun de s'avancer trop loin sans les données de base sur les conditions de vie et de salaire ${ }^{52}$. Piva n'a aucun mal à développer une telle argumentation à Toronto où l'on est bien servi sur le plan statistique, même pour le début du siècle. Il est dommage qu'on n'ait pas encore appliqué ces mêmes techniques aux villes plus petites de l'arrière-pays, où la vaste majorité des Canadiens travaillaient dans les mines et dans la forêt.

Tout comme Piva, Paul Craven travaille dans une perspective marxiste; de la même façon que Kealey, il est sous l'influence d'un historien britannique. Il serait difficile d'imaginer que son ouvrage, An Impartial Umpire, centré sur Mackenzie King et les relations industrielles, puisse être plus éloigné de la «nouvelle» histoire. Même les représentants ouvriers et patronaux y sont des personnages nébuleux alors que les ouvriers ne se situent que dans l'arrière-plan. Néanmoins, si les relations industrielles ne doivent pas occuper l'ensemble de la scène de l'histoire du mouvement ouvrier, l'on ne peut les en exclure complètement. Il faut reconnaître que l'appréciation faite par Craven de King et de l'Industrial Disputes Investigation Act revêt de l'intérêt tout en étant à la fois inattendu et, somme toute, plus sympathique que quiconque ne s'y attendait. Cette appréciation découle peut-être de l'application de la logique de Ralph Miliband exposée dans The State in Capitalist Society ou encore de l'attrait posthume du journal de King, bizarrement convaincant ${ }^{53}$.

Ce qui pour Gene Homel devait être l'étude d'un important personnage du mouvement ouvrier, James Simpson du CMTC, a largement débordé la simple biographie. L'affable typographe-socialiste s'est tissé une vie communautaire tellement riche qu'aucun des travailleurs de Hamilton étudiés par Palmer n'aurait pu se mesurer à lui. Comme le découvrit son biographe, l'ensemble des activités de Simpson au CMTC, au Parti socialiste du Canada, aux sociétés de tempérance, aux loges orangistes, à l'Église méthodiste, à l'Epworth League, au ministère de l'éducation et au Labour Temple, ainsi que ses innombrables autres activités lui laissaient si peu de temps pour gagner sa vie qu'il doit épouser une femme prospère et vivre des rentes des maisons dont elle était propriétaire ${ }^{54}$. Malheureusement les travaux de Homel n'ont pas encore été publiés et son avenir universitaire est incertain.

\footnotetext{
51 Michael Piva, The Condition of the Working Class in Toronto (Ottawa, 1979).

52 Ibid., x; pour la réplique, voir Palmer, «Working Class Canada», 605.

53 Craven, ¿Impartial Umpire», surtout le chap. IX. Voir Ralph Miliband, The State and Capitalist Society (London, 1972).

54 Gene H. Homel, «James Simpson and the Origins of Canadian Social Democracy» (Toronto, Ph.D., 1978).
} 
Mais revenons-en au courant historiographique qui constitue le noyau de l'histoire du mouvement ouvrier au Canada anglais. C'est à juste titre que les historiens de ce courant jouent un rôle clé. Ils ont bien servi l'historiographie canadienne-anglaise à plusieurs égards. Ils se sont installés dans le domaine de façon audacieuse, ils ont mis au défi et provoqué les autres historiens, attiré à leur école quelques étudiants de deuxième et troisième cycles, et parmi les plus brillants, tout en faisant preuve à l'égard des autres historiens d'une plus grande tolérance dans leurs relations personnelles et dans leurs écrits. En jetant un coup d'oeil rétrospectif, on ne considérera peut-être pas le groupe comme le plus important courant historique des années ' 70 - qui oserait se prononcer sur une telle question - mais on pourra ranger ce groupe parmi les mieux documentés. Et pour les historiens, cela suffit peutêtre!

Kealey ayant annoncé que ses récents propos sur l'historiographie seraient ses derniers, cela mérite qu'on y porte attention ${ }^{55}$. Les épées théoriques seront rengainées, au profit des charrues et des émondoirs... Même son collègue le plus terne et le moins enclin à la théorie devrait être en mesure de prendre exemple sur lui.

Pour reprendre le cliché des études historiographiques: il reste beaucoup à faire en histoire des travailleurs. L'on trouvera peut-être des chercheurs pour s'atteler à la tâche. Les études avancées connaissent encore une expansion considérable même si l'on ne sait pas très bien où se trouvent les ressources pour soutenir une telle expansion. Des 1125 thèses de doctorat et de maîtrise en cours en 1981, près du tiers, soit 318, traitaient d'un sujet relevant de l'histoire sociale, et 60 se situaient dans le domaine de l'histoire du mouvement ouvrier ${ }^{56}$. Des recherches qui peuvent paraître très éloignées du domaine peuvent aussi y projeter un éclairage précieux. Récemment une thèse hautement technique sur les mines d'or et de charbon de la Nouvelle-Écosse étudiait de façon marginale les effets bénéfiques et néfastes sur les mineurs du fait que la Provincial Workmen's Association avait réussi à jouer un rôle significatif dans le contrôle du service d'inspection des mines ${ }^{57}$.

On a beaucoup retardé à élargir l'histoire de la classe ouvrière au Canada. Si les historiens de la «deuxième génération»se sont plongés dans la fin du XIX ${ }^{\mathrm{e}}$ siècle, il existe encore de nombreux champs vers lesquels les générations à venir peuvent diriger leurs énergies. Bien entendu, loin d'attribuer des intentions malveillantes à leurs aînés, ou de les accuser de négligence, les historiens de la «deuxième génération» auraient pu avoir la gentillesse de supposer que leurs prédécesseurs

\footnotetext{
55 Kealey, «Labour and Working-Class», 95.

56 Register of Dissertations, XVI, 1981

57 Donald E. MacLeod, «Mining Men, Miners and Mining Reform: Changing the Technology of Nova Scotian Gold Miners and Collieries, 1858-1910» (Toronto, Ph.D., 1981).
} 
avaient tout simplement déblayé un terrain fertile, pour le laisser en jachère en attendant des successeurs plus compétents et plus clairvoyants.

La participation des travailleurs canadiens aux guerres mondiales constitue, par exemple, un domaine qui reste à explorer. Dans le cas de la Première Guerre mondiale, ce sont plus d'un demi-million de Canadiens qui ont vécu l'expérience de la vie militaire, et, dans la Deuxième, ce chiffre s'élève à plus d'un million. Il faut intégrer leur expérience à l'histoire de l'ensemble de la classe ouvrière. Les bonnes intentions et la fragilité qui caractérisent certains projets d'histoire orale ne sauraient suffire ${ }^{58}$. Bill Gammage en Australie ou Dennis Winter en Grande-Bretagne pourraient servir d'exemples ${ }^{59}$. La réintégration des vétérans après chacune des guerres revêt une importance tout aussi grande. Ni le mouvement ouvrier ni ses historiens n'ont saisi jusqu'à quel point la guerre avait réussi à créer un clivage additionnel entre vétérans et non-vétérans au sein d'une classe ouvrière déjà divisée ${ }^{60}$.

En préparant avec Terry Copp, l'introduction au volume Working People, nous avons vite découvert les lacunes de nos connaissances historiques sur l'histoire des travailleurs, du moins en ce qui concerne l'historiographie canadienne-anglaise. Ces lacunes marquent non pas la période que la «deuxième génération» a classée comme la période d'industrialisation, soit de 1860 à 1930 , mais plutôt la période qui a suivi, soit les années quarante et cinquante. Même si ce n'est pas durant cette période que le mouvement syndical a réalisé son unité, il a néanmoins acquis un poids considérable en terme d'effectifs, d'expériences, de changements technologiques, de liens internationaux, d'activités politiques et de mutations socio-culturelles. Comme le soutient mon collègue Terry Copp, il s'agit de la période durant laquelle les Canadiens pauvres, qui représentaient jusqu'alors la majorité numérique de la population, sont devenus une minorité, une minorité, cependant, qui conserve des proportions scandaleuses. De peur qu'une telle affirmation ne soit interprétée comme une hérésie travailliste venant d'une personne qui croit que l'être humain peut, par ses efforts, améliorer son sort, je m'empresse d'ajouter que cette victoire, comme toutes les autres, ne fut que temporaire et éphémère.

Parmi les travaux dignes de mention produits en anglais et portant sur cette période, il y a peu d'études à signaler si ce n'est deux bonnes

\footnotetext{
58 Daphne Read (ed.), The Great War and Canadian Society: An Oral History (Toronto, 1978). En toute honnêteté, le livre n'est pas pire que plusieurs publications récentes à visée commerciale sur le même thème mais les prétentions de Russell Hann dans l'introduction ont pour effet de déprécier ce qu'il y a de valable dans le livre.

59 Bill Gammage, The Broken Years: Australians in the First World War (Canberra, 1974); Dennis Winters, Death's Men (Harmondsworth, 1978).

60 Voir D. Morton et Glenn Wright, «From the Wars Returning: The Bonus Campaign, 1919-21», Canadian Historical Review, LXIV, 2 (June 1983).
} 
thèses, par ailleurs très techniques, consacrées surtout aux relations industrielles: «Remember Kirkland Lake» de Laurel Sefton McDowell et «Technological Change and the Railway Unions» de Rosemary Speirs $^{61}$. La première paraîtra prochainement aux Presses de l'Université de Toronto.

C'est à cette période relativement récente mais en même temps historique qu'on peut encore poser des questions, ces questions auxquelles on ne peut plus et on ne pourra plus jamais répondre pour la période des années 1880 . Si on attend trop longtemps, les éboueurs auront réglé le sort des archives, et les témoins directs de cette période seront ensevelis.

Les historiens de la «deuxième génération» ont promis de produire à un moment donné une nouvelle synthèse de l'histoire canadienne et peut-être même québécoise. Comme, de toute façon, de telles oeuvres se produisent à peu près à toutes les générations, ces derniers sauront sans doute tenir leurs promesses. Le travail effectué par les historiens du mouvement ouvrier durant la dernière décennie, à l'intérieur de collectifs ou non, a déjà transformé de façon sensible notre manière d'aborder les travailleurs, les syndicats et les conflits de classes, de la même façon que ce travail dans un domaine étroitement relié avait modifié notre façon d'envisager la question des femmes. Nous savons maintenant qu'il n'y a pas eu que des radicaux dans les provinces de l'Ouest, que les travailleurs spécialisés jouissaient d'une plus grande autonomie sur les lieux de production qu'ils n'en ont maintenant, et que la ville d'Amherst en Nouvelle-Écosse avait connu sa grève générale en 1919 au même titre que Winnipeg, Toronto, Brandon et Vancouver. De plus, pour peu qu'ils prêtent attention à Jacques Rouillard, les historiens canadiens-anglais en viendront peut-être même à réaliser que le mouvement ouvrier québécois n'a pas attendu qu'un futur premier ministre décide de rendre visite à la ville d'Asbestos pour manifester son militantisme ${ }^{62}$.

Ces facteurs et bien d'autres faits et interprétations ont modifié notre attitude. Nous ont-ils convaincus de jeter notre cargaison à la mer ou d'en disposer autrement? Comme le soulignait le professeur McNaught dans son essai primé sur le sujet, la syndicalisation reste encore le meilleur outil dont les travailleurs canadiens se soient dotés

\footnotetext{
61 Laurel MacDowell, «Remember Kirkland Lake: The Effects of the Kirkland Lake Gold Miners' Strike, 1941-2 (Toronto, Ph.D., 1979) et «The Formation of the Canadian Industrial Relations System During World War II», Labour/Le Travailleur, III (1978); Rosemary Speirs, «Technological Change and the Railway Unions, 1945-1972» (Toronto, Ph.D., 1974).

62 Jacques Rouillard, compte rendu de Fernand Harvey, «Le Mouvement ouvrier au Québec», Revue d' histoire de l'Amérique française, 34.3 (déc. 1980): 452-453. Nolan Reilly, "The General Strike in Amherst, Nova Scotia, 1919» Acadiensis, IX, 2 (printemps 1981). (De tels rappels seront longs à être entendus au Canada anglais). Voir McNaught, «Thompson vs. Logan», 155-156.
} 
pour se protéger. Même les «nouveaux historiens» pourraient être d'accord avec l'affirmation que «les formes et politiques de syndicalisation ont été déterminées par les leaders dont plusieurs sont devenus des 〈collaborateurs〉 ». «La violence, de poursuivre le professeur McNaught ... a (le) plus souvent été provoquée par l'État que par les travailleurs». Pourtant, indépendamment de l'orientation de leurs doléances de classe, les travailleurs canadiens ont constamment eu recours à des grèves nonrévolutionnaires, à des pressions sur les principaux partis politiques et, dans certaines régions et à certains moments, à l'appui à des partis sociaux-démocrates ${ }^{63}$.

Au Québec peut-être autant qu'au Canada anglais, il reste encore beaucoup à faire pour combler les lacunes dans le domaine de nos connaissances historiques. Le temps est maintenant venu de s'attaquer aux problèmes de synthèse si nous ne voulons pas que l'histoire du mouvement ouvrier ne devienne un autre ghetto aussi romantique qu'irrémédiablement dépassé. Cela ne va pas sans rappeler l'éditorial du Toronto Globe des années 1880, qui faisait remarquer qu'autant les syndicats avaient été bénéfiques et même nécessaires dans le passé, autant ils étaient devenus désuets, inutiles et irresponsables. Dans son programme scolaire, l'Ontario fait maintenant figurer l'histoire du mouvement ouvrier avec le vote des femmes dans un bloc intitulé «les réformes sociales». La période couverte s'arrête en 1919. Les éditeurs de manuels nous offrent maintenant une section «histoire du mouvement ouvrier», centrée essentiellement sur l'industrialisation de la fin du XIX $\mathrm{X}^{\mathrm{e}}$ siècle $^{64}$. On ignore systématiquement l'époque la plus significative de la syndicalisation au Canada, les années 1940.

Pour la plupart d'entre nous, l'étude de l'histoire de la classe ouvrière est bien plus qu'une distraction intellectuelle. Si nous étudions l'histoire du mouvement ouvrier, c'est bien pour en changer le cours. Comme d'habitude au Canada anglais, nous courons le risque d'être détournés de notre objectif.

63 Ibid., 167-168.

64 À titre d'exemple, R. Douglas Francis et Donald B. Smith, Readings in Canadian History: Post-Confederation (Toronto, 1982), 155-204. 